\title{
Patients Undergoing Total Shoulder Arthroplasty on the Dominant Extremity Attain Greater Postoperative ROM
}

\author{
Gregory L. Cvetanovich MD, Peter N. Chalmers MD, Jonathan J. Streit MD, \\ Anthony A. Romeo MD, Gregory P. Nicholson MD
}

Received: 7 February 2015/Accepted: 3 June 2015/Published online: 12 June 2015

(C) The Association of Bone and Joint Surgeons \& 2015

\begin{abstract}
Background Total shoulder arthroplasty (TSA) provides excellent functional outcomes and pain relief in appropriately selected patients. Although it is known to affect other shoulder conditions, the role of hand dominance after TSA has not been reported, to our knowledge.

Questions/Purposes We asked: (1) Does TSA of the dominant arm result in greater postoperative ROM
\end{abstract}

The institution of one or more of the authors (AAR and GPN) has received, during the study period, research funding from Arthrex, Smith and Nephew, and Donjoy.

One of the authors certifies that he (AAR) has received or may receive payments or benefits, during the study period, an amount of USD $\$ 100,001$ to $\$ 1,000,000$ from Arthrex, Inc, Naples, FL, USA).

One of the authors certifies that he (GPN) has received or may receive payments or benefits, during the study period, an amount of USD

$\$ 10,001$ to $\$ 100,000$ from Tornier, Inc, Bloomington, MN, USA), and an amount less than USD \$10,000 from Innomed, Inc (Savannah, GA, USA).

All ICMJE Conflict of Interest Forms for authors and Clinical Orthopaedics and Related Research ${ }^{\circledR}$ editors and board members are on file with the publication and can be viewed on request.

Clinical Orthopaedics and Related Research ${ }^{\mathbb{R}}$ neither advocates nor endorses the use of any treatment, drug, or device. Readers are encouraged to always seek additional information, including FDA approval status, of any drug or device before clinical use.

Each author certifies that his or her institution approved or waived approval for the reporting of this investigation and that all investigations were conducted in conformity with ethical principles of research.

This work was performed at Rush University Medical Center.

G. L. Cvetanovich ( $₫)$, P. N. Chalmers, A. A. Romeo,

G. P. Nicholson

Section of Shoulder and Elbow Surgery, Department of

Orthopaedic Surgery, Rush University Medical Center, 1611 W.

Harrison Street, Suite 201, Chicago, IL 60612, USA

e-mail: Gregory.cvetanovich@gmail.com compared with TSA of the nondominant arm? (2) Does hand dominance affect validated outcome scores after TSA?

Methods We performed a review of all patients who underwent primary TSAs between 2008 and 2011 with a minimum of 12 months followup. During that time, one surgeon performed 156 primary TSAs. One hundred twenty-seven patients met the minimum followup requirement for this analysis (81\%), whereas 29 (19\%) were lost to followup. Seven patients were excluded for surgical indications other than glenohumeral osteoarthritis. A total of 58 patients underwent TSA of the dominant upper extremity and 62 underwent TSA of the nondominant upper extremity. Patient demographics, preoperative and postoperative ROM, and preoperative and postoperative outcome scores, were collected from the medical records. Student's t-tests and chi-square tests were used for analysis. Demographics and preoperative ROM did not differ between patients undergoing TSA on the dominant or the nondominant upper extremity.

Results Dominant-arm TSAs showed greater postoperative forward elevation and external rotation. Postoperative active forward elevation in the dominant group was $151^{\circ}$ versus $141^{\circ}$ in the nondominant group (mean difference, $\left.10^{\circ} ; 95 \% \mathrm{CI}, 1^{\circ}-18^{\circ} ; \mathrm{p}=0.033\right)$. Postoperative active external rotation was $61^{\circ}$ in the dominant group, versus $51^{\circ}$ in the nondominant group (mean difference, $10^{\circ} ; 95 \% \mathrm{CI}$, $\left.5^{\circ}-15^{\circ} ; \mathrm{p}<0.001\right)$. Active internal rotation did not differ (dominant, $52^{\circ}$, nondominant, $50^{\circ}$; mean difference, $2^{\circ}$;

\section{J. J. Streit}

Department of Orthopaedics, Case Western Reserve University, Cleveland, OH, USA 
$95 \% \mathrm{CI},-3^{\circ}$ to $\left.7^{\circ} ; \mathrm{p}=0.419\right)$. There were no differences in postoperative VAS (dominant, 0.9, nondominant, 1.4; mean difference, $0.5 ; 95 \% \mathrm{CI},-0.1$ to $1.1 ; \mathrm{p}=0.129$ ), simple shoulder test (dominant, 9.8, nondominant, 9.2; mean difference, $0.5 ; 95 \% \mathrm{CI},-0.5$ to $1.5 ; \mathrm{p}=0.278$ ), and American Shoulder and Elbow Surgeons scores (dominant, 84 , nondominant, 80 ; mean difference, $4 ; 95 \% \mathrm{CI},-2$ to $10 ; \mathrm{p}=0.211$ )

Conclusions Patients who underwent TSA of their dominant upper extremity had greater postoperative active forward elevation and active external rotation compared with patients who had TSA of their nondominant upper extremity. This average difference of $10^{\circ}$ active forward elevation and active external rotation could be useful for preoperative and postoperative counseling of patients. Regardless of hand dominance, similar functional outcomes were achieved.

Level of Evidence Level III, therapeutic study.

\section{Introduction}

Total shoulder arthroplasty (TSA) is an effective treatment for patients with degenerative joint disease of the glenohumeral joint in the setting of a functional rotator cuff, providing functional improvement and pain relief $[5,14,18]$. Although concerns persist regarding complications such as glenoid loosening [15], the number of TSAs performed in the United States has been increasing [1,8]. The cost-effective TSA also has been shown to improve patient function, pain outcome scores, and overall physical well-being $[3,11]$.

Hand dominance has been reported to affect numerous shoulder disorders including anterior glenohumeral instability [6], rotator cuff disorders [9], and shoulder disorders in the throwing athlete [7]. Hand dominance also has been reported to affect the minimum clinically important difference in patient-reported VAS for rotator cuff disease [22]. In addition, hand dominance affects scapular and clavicular kinematics in nonthrowing patients and kinematics and kinetics in throwing athletes [12, 13, 23]. There are limited and conflicting data available in the shoulder arthroplasty literature regarding the effect of hand dominance on outcome after TSA $[2,4,16,18,20,21]$. Information regarding potential differences in TSA outcomes based on hand dominance could be used for patient counseling about expected outcomes. In addition, this factor may need to be considered in future comparative studies with ROM as an outcome.

Therefore, we asked: (1) Does TSA of the dominant arm result in greater postoperative ROM compared with TSA of the nondominant arm? (2) Does hand dominance affect outcome scores after TSA?

\section{Methods}

This study was approved by our institutional review board. The study design was a retrospective case series. All patients undergoing primary anatomic TSA by the senior author (GPN) between January 1, 2008 and October 1, 2011, with a minimum of 12 months postoperative followup, were identified for potential inclusion in the study.

Patients were excluded for prior ipsilateral shoulder arthroplasty, reverse TSA, and incomplete postoperative records. Only patients undergoing a TSA for glenohumeral osteoarthritis with an intact rotator cuff were included. Patients who had a TSA for posttraumatic glenohumeral arthritis, glenohumeral instability arthropathy, or other indications were excluded.

We retrospectively reviewed patient records, including operative reports, preoperative office notes, and postoperative office notes. We also extracted the following data for each patient from the medical records: age, sex, laterality of dominant upper extremity, laterality of TSA, and preoperative and postoperative ROM at most recent followup.

ROM measurements were obtained by the senior surgeon with a goniometer in his routine preoperative and postoperative physical examinations. Validated outcome scores from the VAS for pain, simple shoulder test (SST) [10], and the American Shoulder and Elbow Surgeons (ASES) score [19] were obtained before surgery and at the most recent followup.

During the study period, one surgeon (GPN) performed 156 primary TSAs in 156 patients. Of those, 127 patients met the minimum followup requirement for this analysis (81\%), whereas $29(19 \%)$ were lost to followup. Seven patients were excluded for surgical indications other than glenohumeral osteoarthritis. As a result, we included 120 patients for further analysis, of whom $48 \%$ were female with an average age of 67 years. Followup was a mean of 23 months after surgery (range, 12-108 months).

Of the 120 included patients, 58 underwent TSA of the dominant upper extremity, of whom 55 were right-hand-

Table 1. Patient demographics

\begin{tabular}{llll}
\hline Demographic & Dominant & Nondominant & p value \\
\hline Number of patients & 58 & 62 & NA \\
Age (years) (mean \pm SD) & $66.0+/-9.1$ & $67.3+/-10.2$ & 0.464 \\
\% female & 43.1 & 53.2 & 0.279 \\
Hand dominance & & & 0.493 \\
Right & 55 & 56 & \\
Left & 3 & 6 & \\
\hline
\end{tabular}

$\mathrm{NA}=$ not applicable. 
dominant and three were left-hand-dominant. Conversely, 62 patients underwent TSA of the nondominant upper extremity, of whom 56 were right-hand-dominant and six were left-hand-dominant. Patient demographics were not statistically different between the two groups (Table 1). Preoperative ROM and functional scores also were not statistically different between the two groups (Table 2).

Statistical analyses were performed using SPSS Version 18 (SPSS Inc, Chicago, IL, USA). Descriptive statistics were calculated. Kolmogorov-Smirnov analysis was performed to confirm data normality. Based on data normality, groups were compared using Student's t-tests with significance set at $p$ less than 0.05 . Chi-square tests were used for statistical comparison of categorical variables with significance set at $\mathrm{p}$ less than 0.05 .

\section{Results}

Patients undergoing dominant-arm TSA showed greater postoperative active forward elevation and external rotation. Postoperative active forward elevation in the dominant group was $151^{\circ}$, which was greater than $141^{\circ}$ in the nondominant group (mean difference, $10^{\circ} ; 95 \% \mathrm{CI}, 1^{\circ}-$ $18^{\circ} ; \mathrm{p}=0.033$ ). Postoperative active external rotation in the dominant group was $61^{\circ}$, which was greater than $51^{\circ}$ in the nondominant group (mean difference, $10^{\circ} ; 95 \% \mathrm{CI}, 5^{\circ}$ $15^{\circ} ; \mathrm{p}<0.001$ ) (Fig. 1). There was no difference between the dominant and nondominant groups in active internal rotation postoperatively (dominant, $52^{\circ}$, nondominant, $50^{\circ}$; mean difference, $2^{\circ} ; 95 \% \mathrm{CI},-3^{\circ}$ to $7^{\circ} ; \mathrm{p}=0.419$ ) (Table 3).

Comparing VAS (dominant, 0.9, nondominant, 1.4; mean difference, $0.5 ; 95 \% \mathrm{CI},-0.1$ to $1.1 ; \mathrm{p}=0.129$ ), SST (dominant, 9.8, nondominant, 9.2; mean difference, 0.5; 95\% CI, -0.5 to $1.5 ; \mathrm{p}=0.278$ ), and ASES scores (dominant, 84, nondominant, 80; mean difference, 4; 95\% CI, -2 to $10 ; p=0.211$ ), there were no differences between outcome scores for hand dominance effect after TSA (Table 3). A post hoc power analysis based on ASES scores found that our study has a power of 0.39 , leaving it underpowered on this endpoint. To achieve a power of 0.8 , we would have needed 197 patients per group (197 dominant and 197 nondominant, for a total sample size of 394).

\section{Discussion}

TSA is an increasingly common procedure that improves function and relieves pain for patients with degenerative joint disease of the glenohumeral joint in the setting of a functional rotator cuff $[3,5,11,14,17,18]$. Although hand dominance has been shown to affect shoulder disorders including anterior glenohumeral instability [6], rotator cuff disorders [9], and shoulder disorders in the throwing athlete [7], there are limited and conflicting data regarding the role of hand dominance in TSA outcome $[2,4,16,18,20,21]$.

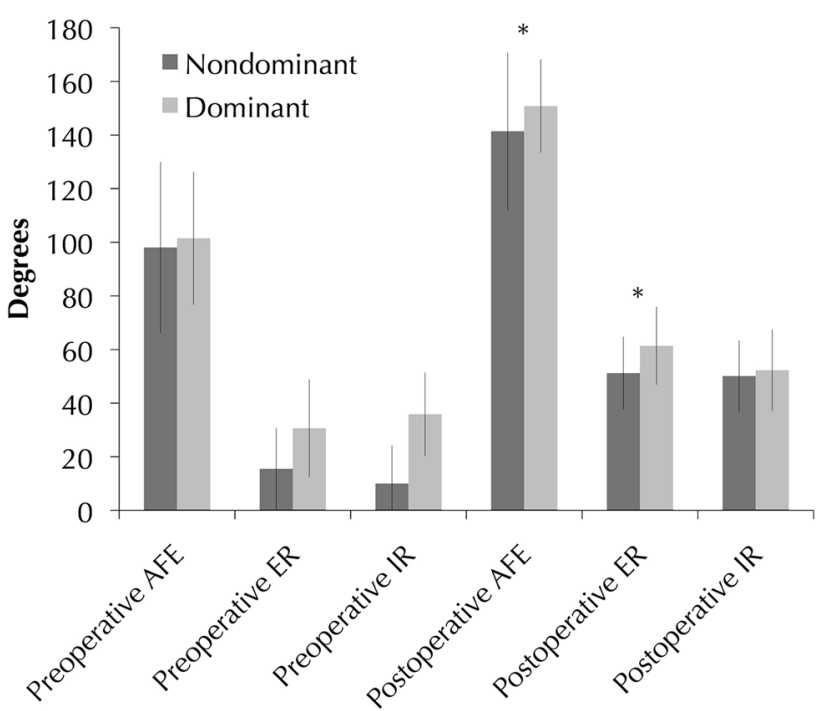

Fig. 1 Greater postoperative active forward elevation and active external rotation was seen in patients who had TSA of the dominant extremity versus patients who had TSA of the nondominant upper extremity. AFE = active forward elevation; $\mathrm{ER}=$ external rotation; IR $=$ internal rotation. There were significant differences between dominant and nondominant groups for postoperative active forward elevation $(\mathrm{p}=0.033)$ and external rotation $(\mathrm{p}<0.001)$.

Table 2. Preoperative ROM and outcome in dominant and nondominant upper extremity groups

\begin{tabular}{|c|c|c|c|c|}
\hline Preoperative findings & Dominant & Nondominant & Mean difference & $\mathrm{p}$ value \\
\hline Preoperative active forward elevation & $102(91-112)$ & $98(83-113)$ & $3(-15$ to 22$)$ & 0.711 \\
\hline Preoperative active external rotation & $31(18-43)$ & $16(6-25)$ & $15(-2$ to 32$)$ & 0.072 \\
\hline Preoperative active internal rotation & $36(23-48)$ & $10(-10$ to 30$)$ & $26(-5$ to 57$)$ & 0.086 \\
\hline Preoperative VAS & $5.2(4.6-5.8)$ & $5.4(4.7-6.1)$ & $0.2(-0.7$ to 1.1$)$ & 0.613 \\
\hline Preoperative simple shoulder test & $4.3(3.6-5)$ & $3.9(3.2-4.6)$ & $0.4(-0.6$ to 1.4$)$ & 0.435 \\
\hline Preoperative ASES & $45(40-49)$ & $40(34-45)$ & $5(-2$ to 12$)$ & 0.145 \\
\hline
\end{tabular}

ASES = American Shoulder and Elbow Surgeons; all values are reported as mean and 95\% CI. 
Table 3. Postoperative ROM and outcome scores

\begin{tabular}{llllr}
\hline Postoperative findings & Dominant & Nondominant & Mean difference & $\mathrm{p}$ value \\
\hline Postoperative active forward elevation & $151(146-155)$ & $141(134-149)$ & $9(1-18)$ & 0.033 \\
Postoperative active external rotation & $61(58-65)$ & $51(48-55)$ & $10(5-15)$ & $2(-3$ to 8$)$ \\
Postoperative active internal rotation & $52(48-56)$ & $50(47-54)$ & $0.5(-0.1$ to 1.1$)$ & 0.419 \\
Postoperative VAS & $0.9(0.6-1.3)$ & $1.4(0.9-1.9)$ & $0.5(-0.4$ to 1.5$)$ & 0.129 \\
Postoperative simple shoulder test & $9.8(9.2-10.3)$ & $9.2(8.5-10)$ & $4(-2$ to 10$)$ & 0.278 \\
Postoperative ASES & $84(81-88)$ & $80(76-85)$ & 0.211 \\
\hline
\end{tabular}

ASES = American Shoulder and Elbow Surgeons; all values are reported as mean and 95\% CI.

Patients who underwent TSA of their dominant upper extremity had greater postoperative active forward elevation and active external rotation compared with patients who underwent TSA of their nondominant upper extremity. We did not find a difference in outcomes scores. Clinicians may find this information a valuable addition to preoperative counseling, particularly for patients who are undergoing bilateral TSAs. In addition, future TSA studies should consider the variable of hand dominance, which may influence the amount of improvement in pain and function that can be expected after surgery, to validate or refute our findings.

Our study does have limitations, including potential unmeasured confounding variables, relatively short followup, and retrospective nature. Additionally, there is potential assessor bias as only one observer (GPN) did ROM measurements and there was no blinding with respect to hand dominance and analysis of ROM. We think hand dominance is unlikely to bias our ROM data acquisition. Our patients had relatively short followup with a minimum of 12 months and an average of 23 months. This minimum followup was thought to allow patients to reach full ROM recovery from the surgery. In our clinical experience, ROM in patients does not improve to any great degree after the first postoperative year. However, longer-term followup studies might address whether the difference in ROM based on hand dominance persists. During the study period, the followup for patients who had TSAs performed by one surgeon (GPN) was $81 \%$, with only $19 \%$ of patients lost to followup. It is possible that the patients lost to followup had inferior outcomes to those who returned, but given the high rate of followup, any bias related to this would be relatively small. In addition, this study may be subject to Type II error based on failure to identify a difference in outcome scores between the dominant and nondominant groups based on insufficient power.

We found that arm dominance was associated with postoperative ROM after TSA, but no difference in outcomes based on arm dominance. With the numbers available, we were underpowered to detect such differences in outcomes, and it is possible that our study could have failed to detect a clinically important difference in that regard. However, some studies [2, 4, 16, 18, 20, 21] have considered arm dominance as it affects clinical outcomes, but to our knowledge, none has identified an effect of arm dominance on postoperative ROM. Brostrom et al. [2] reported an early retrospective series of 16 patients with rheumatoid arthritis who had TSAs, stating that "patients who had a TSA performed on their dominant side were less satisfied with the outcome than those operated on the nondominant side." They did not provide additional data or statistical analysis regarding this. Raiss et al. [18] analyzed long-term retrospective outcomes of TSAs in 63 patients at 15 to 20 years after surgery, finding that hand dominance was not associated with an inferior clinical outcome for patients who had not undergone revision. Collin et al. [4] conducted a prospective cohort study of 56 patients who had TSAs and with 10 years followup, showing that TSA of the dominant upper extremity is a significant factor predicting progression of radiolucent lines of the glenoid component after TSA. Several other studies reported no association of radiolucent lines and glenoid component loosening with arm dominance [16, 20, 21].

In our opinion, an average $10^{\circ}$ greater active forward elevation and active external rotation for TSA of the dominant versus nondominant upper extremity is not only statistically significant but also clinically significant for patients who had TSAs. To our knowledge, there are no published minimum clinically important differences for shoulder ROM, and this would be a subject for further study to determine if a $10^{\circ}$ difference in forward elevation and external rotation is clinically important. The gain in active forward elevation may be particularly clinically significant as patients participate in overhead activities. Patients can be counseled that on average they will achieve slightly greater active forward elevation and active external rotation in the dominant than in the nondominant extremity after a TSA. This finding also might help surgeons answer a common postoperative clinical question from patients with bilateral TSAs: "Why is my right shoulder better than my left?"

Patients who underwent a TSA of their dominant upper extremity had greater postoperative active forward 
elevation and active external rotation compared with patients who had a TSA of their nondominant upper extremity. The difference of $10^{\circ}$ active forward elevation and active external rotation could be useful for preoperative and postoperative counseling, although future study is needed to determine if these differences exceed the minimum clinically important difference for shoulder ROM. Regardless of hand dominance, no differences in functional outcomes were achieved.

\section{References}

1. Adams JE, Sperling JW, Hoskin TL, Melton LJ 3rd, Cofield RH. Shoulder arthroplasty in Olmsted County, Minnesota, 1976-2000: a population-based study. J Shoulder Elbow Surg. 2006;15:50-55.

2. Broström LA, Wallensten R, Olsson E, Anderson D. The Kessel prosthesis in total shoulder arthroplasty: a five-year experience. Clin Orthop Relat Res. 1992;277:155-160.

3. Carter MJ, Mikuls TR, Nayak S, Fehringer EV, Michaud K. Impact of total shoulder arthroplasty on generic and shoulderspecific health-related quality-of-life measures: a systematic literature review and meta-analysis. $J$ Bone Joint Surg Am. 2012;94:e127.

4. Collin P, Tay AK, Melis B, Boileau P, Walch G. A ten-year radiologic comparison of two-all polyethylene glenoid component designs: a prospective trial. J Shoulder Elbow Surg. 2011;20:1217-1223.

5. Deshmukh AV, Koris M, Zurakowski D, Thornhill TS. Total shoulder arthroplasty: long-term survivorship, functional outcome, and quality of life. J Shoulder Elbow Surg. 2005;14:471-479.

6. Edouard P, Degache F, Beguin L, Samozino P, Gresta G, FayolleMinon I, Farizon F, Calmels P. Rotator cuff strength in recurrent anterior shoulder instability. J Bone Joint Surg Am. 2011;93:759-765.

7. Hibberd EE, Oyama S, Myers JB. Increase in humeral retrotorsion accounts for age-related increase in glenohumeral internal rotation deficit in youth and adolescent baseball players. Am J Sports Med. 2014;42:851-858.

8. Jain NB, Higgins LD, Guller U, Pietrobon R, Katz JN. Trends in the epidemiology of total shoulder arthroplasty in the United States from 1990-2000. Arthritis Rheum. 2006;55:591-597.

9. Keener JD, Steger-May K, Stobbs G, Yamaguchi K. Asymptomatic rotator cuff tears: patient demographics and baseline shoulder function. J Shoulder Elbow Surg. 2010;19:1191-1198.

10. Lippitt SB, Harryman DT II, Matsen FA 3rd. A practical tool for evaluating function: the Simple Shoulder Test. In: Matsen FA, Fu FH, Hawkins RJ, eds. The Shoulder: A Balance of Mobility and Stability. Rosemont, IL: American Academy of Orthopaedic Surgeons; 1993:501-518.
11. Mather RC 3rd, Watters TS, Orlando LA, Bolognesi MP, Moorman CT 3rd. Cost effectiveness analysis of hemiarthroplasty and total shoulder arthroplasty. $J$ Shoulder Elbow Surg. 2010;19:325-334.

12. Matsuki $\mathrm{K}$, Matsuki $\mathrm{KO}, \mathrm{Mu} \mathrm{S}$, Kenmoku T, Yamaguchi S, Ochiai N, Sasho T, Sugaya H, Toyone T, Wada Y, Takahashi K, Banks SA. In vivo 3D analysis of clavicular kinematics during scapular plane abduction: comparison of dominant and nondominant shoulders. Gait Posture. 2014;39:625-627.

13. Matsuki K, Matsuki KO, Mu S, Yamaguchi S, Ochiai N, Sasho T, Sugaya $\mathrm{H}$, Toyone T, Wada Y, Takahashi K, Banks SA. In vivo 3-dimensional analysis of scapular kinematics: comparison of dominant and nondominant shoulders. J Shoulder Elbow Surg. 2011;20:659-665.

14. Montoya F, Magosch P, Scheiderer B, Lichtenberg S, Melean P, Habermeyer P. Midterm results of a total shoulder prosthesis fixed with a cementless glenoid component. J Shoulder Elbow Surg. 2013;22:628-635.

15. Papadonikolakis A, Neradilek MB, Matsen FA 3rd. Failure of the glenoid component in anatomic total shoulder arthroplasty: a systematic review of the English-language literature between 2006 and 2012. J Bone Joint Surg Am. 2013;95:2205-2212.

16. Pfahler M, Jena F, Neyton L, Sirveaux F, Molé D. Hemiarthroplasty versus total shoulder prosthesis: results of cemented glenoid components. J Shoulder Elbow Surg. 2006;15:154-163.

17. Radnay CS, Setter KJ, Chambers L, Levine WN, Bigliani LU, Ahmad CS. Total shoulder replacement compared with humeral head replacement for the treatment of primary glenohumeral osteoarthritis: a systematic review. J Shoulder Elbow Surg. 2007; 16:396-402.

18. Raiss P, Bruckner T, Rickert M, Walch G. Longitudinal observational study of total shoulder replacements with cement: fifteen to twenty-year follow-up. J Bone Joint Surg Am. 2014;96:198-205.

19. Richards RR, An KN, Bigliani LU, Friedman RJ, Gartsman GM, Gristina AG, Iannotti JP, Mow VC, Sidles JA, Zuckerman JD. A standardized method for the assessment of shoulder. function. $J$ Shoulder Elbow Surg 1994;3:347-352.

20. Sperling JW, Cofield RH, O'Driscoll SW, Torchia ME, Rowland CM. Radiographic assessment of ingrowth total shoulder arthroplasty. J Shoulder Elbow Surg. 2000;9:507-513.

21. Szabo I, Buscayret F, Edwards TB, Nemoz C, Boileau P, Walch G. Radiographic comparison of flat-back and convex-back glenoid components in total shoulder arthroplasty. J Shoulder Elbow Surg. 2005;14:636-642.

22. Tashjian RZ, Deloach J, Porucznik CA, Powell AP. Minimal clinically important differences (MCID) and patient acceptable symptomatic state (PASS) for visual analog scales (VAS) measuring pain in patients treated for rotator cuff disease. $J$ Shoulder Elbow Surg. 2009;18:927-932.

23. Werner SL, Guido JA, Delude NA, Stewart GW, Greenfield JH, Meister K. Throwing arm dominance in collegiate baseball pitching: a biomechanical study. Am J Sports Med. 2010;38:1606-1610. 